\title{
Uso de obturadores quirúrgicos en cirugía de resección maxilar. Serie de cinco casos y propuesta de diseños
}

\author{
Use of immediate obturators in maxillary resection surgery. \\ Five case series and obturators design proposal
}

\author{
Jorge Sebastián Salinas González, ${ }^{*}$ René Rosales Reyna, ${ }^{+}$Luis Medrano Caballero ${ }^{\S}$
}

\section{RESUMEN}

Los obturadores quirúrgicos o inmediatos son prótesis intraorales utilizadas para mejorar las condiciones postquirúrgicas de los pacientes sometidos a cirugía de resección maxilar por diferentes tipos de neoplasias, ya sean benignas o malignas; se utilizan para mejorar el habla y la respiración, ayudan al proceso de cicatrización del defecto resultante, sirven de barrera entre las cavidades nasal y bucal evitando el paso de alimento hacia la vía respiratoria superior, disminuyen el riesgo de broncoaspiración y el impacto psicológico del paciente, mejoran el soporte de los tejidos blandos faciales evitando retracciones y cicatrizaciones que afectarán la estética a mediano plazo. Otro aporte importante es reducir el tiempo de hospitalización al suprimir la necesidad de colocar sondas de alimentación nasogástricas o de gastrostomía, lo que además afecta de forma importante la calidad de vida del paciente. El uso de estos aditamentos debe ser bien conocido entre los profesionales de la medicina dedicados al área de cirugía de cabeza y cuello. El objetivo de este trabajo es el de presentar cinco casos clínicos de pacientes sometidos a diferentes tipos de resección maxilar como

\section{ABSTRACT}

Immediate obturators are intraoral prosthesis used to improve post-surgical conditions from patients underwent maxillary resections surgeries as treatment of benign or malignant tumors; they're used to improve speech and breath, also help to healing the maxillary defect, as well as a barrier between buccal and nasal cavities avoiding the passage of food to the upper airway, decreases the risk of bronchoaspiration, helps lessen the psychological impact of maxillary resection defects and improves the support of the facial tissues, minimizes scar contracture and disfigurement, bringing a positive effect on the patient's facial aesthetics. It helps reducing hospitalization time by not having the necessity to use a feeding tube, either nasogastric or gastrotomy tubes, which also affects their quality of life. The use of this prosthesis must be know by all head and neck surgeons. The objective of this paper is to present five clinical cases of malignant tumor maxillary resection surgery patients, where immediate obturators were placed as part of an integrated treatment; in addition to proposing different designs of immediate obturators to be used in each particular case. The use of

* Protesista Maxilofacial, Catedrático en la Facultad de Estomatología de la Benemérita Universidad Autónoma de Puebla. México.

‡ Cirujano Maxilofacial, adscrito al Servicio de Cirugía Maxilofacial del Centro Médico Nacional «Manuel Ávila Camacho», IMSS. Puebla, Puebla. México. $\S$ Cirujano Dentista, Facultad de Odontología de la Universidad Popular Autónoma del Estado de Puebla. México. 
tratamiento de neoplasias malignas, donde se colocó un obturador quirúrgico como parte del tratamiento; así como proponer diferentes diseños de los mismos para adecuarlos a cada caso en particular. El uso de obturadores quirúrgicos en cirugía de resección maxilar, debe ser imperativo para mejorar las secuelas propias del procedimiento, así como las secuelas futuras que se pudieran presentar; al mismo tiempo, es importante su uso pensando en la rehabilitación a largo plazo de las funciones orales para mejorar la calidad de vida de los pacientes.

Palabras clave: Obturador quirúrgico, maxilectomía, prótesis maxilofacial, cirugía maxilofacial.

\section{INTRODUCCIÓN}

En la actualidad, las neoplasias del labio y cavidad oral representan un 2.0\% (354,864 pacientes) de los pacientes con cáncer en el ámbito mundial, ${ }^{1}$ en México representan el $6 \%$ del total de las neoplasias malignas en hombres, y un $1.6 \%$ en mujeres. ${ }^{2}$ En el caso de neoplasias del maxilar, el tratamiento quirúrgico de elección es una maxilectomía cuya extensión dependerá de la localización, el tamaño y las características histopatológicas, ${ }^{3,4}$ la cual puede ser total (resección de las cinco paredes del seno maxilar), subtotal superior (resección de cuatro paredes del seno maxilar con preservación del paladar), subtotal inferior (resección de cuatro paredes del seno maxilar con preservación del piso orbitario) y medial (resección de la pared medial del seno maxilar, con extensiones al piso de la órbita y las celdillas etmoidales). ${ }^{5}$

Los defectos postquirúrgicos del maxilar dejan al paciente con una comunicación oroantral que lo predisponen a un habla hipernasal, ${ }^{3,6,7}$ un paso de alimentos y líquidos hacia la cavidad nasal, una masticación comprometida y disfagia. . $, 8,9$ Se presenta una limitación en la apertura oral por cicatrización postquirúrgica, trismus y fibrosis de los músculos de la masticación, principalmente cuando se recibe radioterapia adyuvante. . $^{, 10,11}$

Un obturador es una prótesis maxilofacial que se usa para el cierre de defectos congénitos o adquiridos, principalmente del paladar duro y estructuras contiguas como el reborde alveolar y tejidos blandos. ${ }^{12}$

La literatura histórica menciona que el uso de los obturadores se ha documentado desde el siglo XIV, haciendo referencia a su uso desde mucho tiempo antes. El reporte más significativo en esta área fue realizado por Ambroise Paré (1510-1590), cirujano immediate obturators in maxillary resection it's required to improve the outcome of the surgical procedures, as well as future alterations that may appear. Its use is also important due to the benefits it presents in a possible long term rehabilitation of the oral functions to improve the patient's quality of life.

Keywords: Immediate obturators, maxillectomy, maxillofacial prostheses, maxillofacial surgery.

francés al que se le debe el uso de la palabra obturateurs, la cual deriva de la palabra latina obturo que significa tapar. ${ }^{13}$

Claude Martin, en 1876, describió el uso de un obturador protésico quirúrgico, y en 1927 Fry tomó impresiones en el momento inmediato tras la cirugía. ${ }^{14}$

La secuencia de tratamiento incluye la colocación de un obturador quirúrgico o inmediato, realizado a base de una impresión preoperatoria, el cual se coloca durante o inmediatamente después de la cirugía de resección, éste no debe retirarse en un periodo aproximado de 7-10 días para que cumpla sus funciones principales. Después se colocará un obturador interino o transicional posterior a la cicatrización inicial de la herida, el cual ayuda a promover la continuidad del cierre del defecto. Por último, el obturador definitivo se coloca de seis a 12 meses después de la cirugía, una vez que la enfermedad se encuentre en remisión, que ya no haya cambios en los tejidos blandos y que el equipo quirúrgico y médico dé la autorización para el procedimiento protésico. ${ }^{7,12,15,16}$

Los obturadores quirúrgicos se subclasifican en obturadores quirúrgicos inmediatos y obturadores quirúrgicos tardíos. Los primeros son colocados de manera intraoral durante la cirugía de resección, con la finalidad de restaurar y mantener la función oral durante el periodo postoperatorio, brindar soporte a los tejidos blandos intra y extraorales, favorecer la cicatrización, disminuir las deformaciones faciales, proveer de una separación entre la cavidad nasal y bucal impidiendo el intercambio de secreciones nasales y bucales, mejorar la higiene oral postoperatoria, mejorar las condiciones de fonación, disminuir el impacto psicológico de la pérdida de estructura maxilar y proteger el empaque quirúrgico de restos de alimentos, reduciendo la posibilidad de una infección 
postoperatoria. Los obturadores quirúrgicos tardíos se colocan días después de haber realizado la cirugía, éstos son fabricados con base en una impresión postoperatoria del defecto, por lo que son más exactos. ${ }^{8,9,17}$

Las ventajas que proveen el uso de los obturadores son la restauración de la deglución, la masticación y la fonación, proveer de protección y soporte a los tejidos, impidiendo la contracción de los mismos, el componente dental del obturador permite al paciente masticar alimentos suaves y mejorar la estética, lo cual sirve como estímulo de la moral o terapia psicológica, prevé que la lengua del paciente explore el área del defecto, lo cual impide que esté consciente de las dimensiones reales del mismo y mejora el control de las secreciones orales. ${ }^{11,18-22}$

Una desventaja que se puede llegar a presentar es un ligero desajuste del obturador quirúrgico inmediato debido a su realización previa a la maxilectomía, impidiendo un buen sellado del defecto y permitiendo el paso de líquido hacia la cavidad nasal, lo cual se puede solucionar con el uso de materiales acondicionadores de tejidos. ${ }^{8}$

Las consecuencias de la falta de uso de un obturador postquirúrgico conllevan a una pérdida funcional grave, además de una falta de soporte de los tejidos blandos, provocando contracturas de los mismos, evidenciando una asimetría en el contorno facial. ${ }^{19,20,23}$

\section{CASOS CLÍNICOS}

Se presenta una serie de casos clínicos que ejemplifican el uso de diferentes diseños de obturadores quirúrgicos en diversas cirugías de resección maxilar, sugiriendo un diseño para cada caso y enfatizando su utilidad para disminuir secuelas postoperatorias, lo que ayudará a la futura rehabilitación del paciente.

\section{Caso 1}

Hombre de 38 años con diagnóstico de estesioneuroblastoma de alto grado de cavidad nasal, con infiltración al seno frontal, fosa craneal anterior, cavidad orbitaria y seno maxilar izquierdos. Después de la protocolización del caso, se programa para resección quirúrgica mediante un abordaje craneofacial combinado, mediante craneotomía por abordaje coronal para acceder al tumor de la base de cráneo y hemimaxilectomía izquierda para abordar el tumor en el seno maxilar. El equipo de prótesis maxilofacial
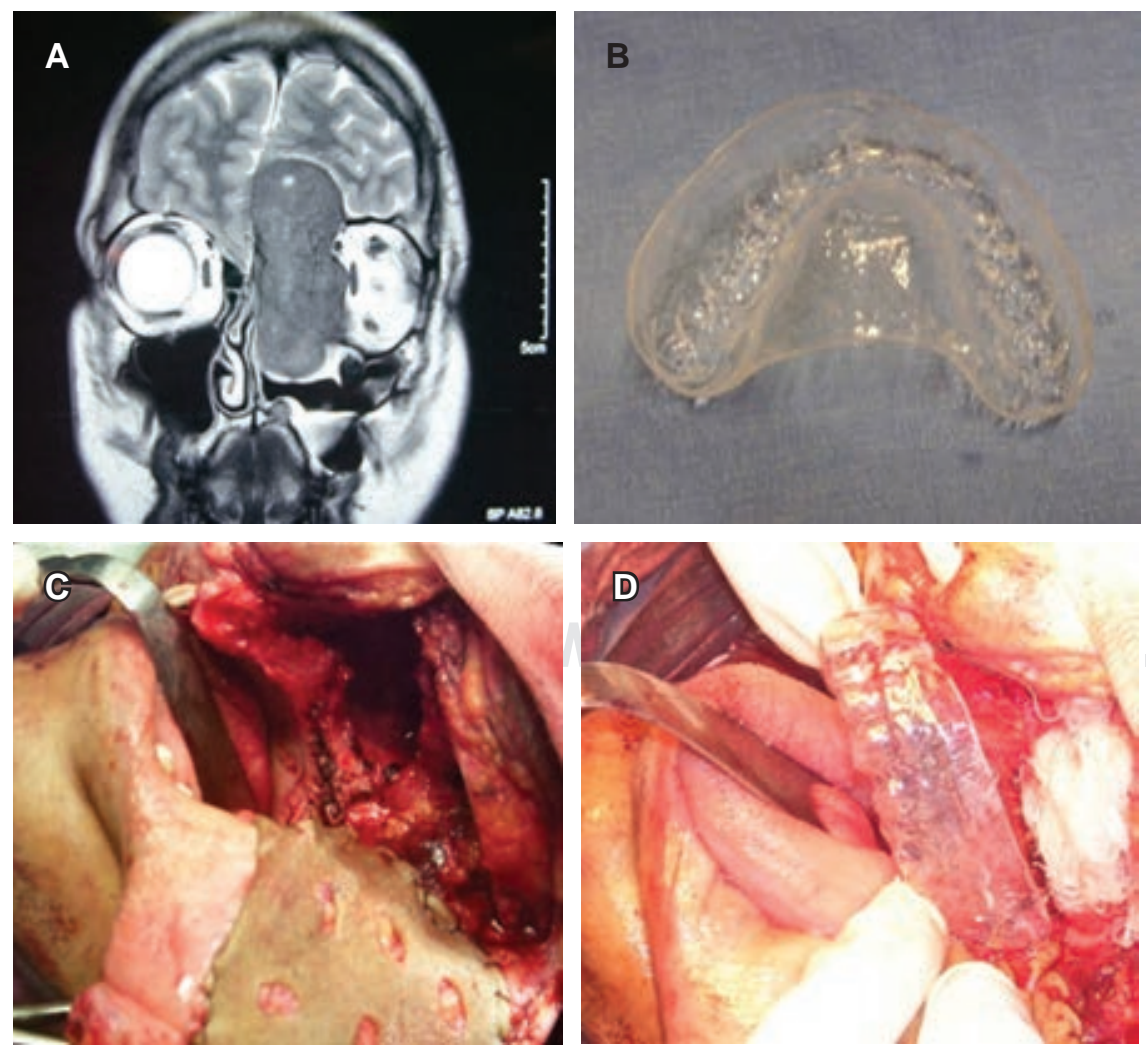

Figura 1:

Caso 1. A) Imagen de resonancia magnética coronal de cavidad nasal y senos paranasales que muestra lesión tumoral (estesioneuroblastoma) que ocupa cavidad nasal, seno maxilar, órbita y fosa craneal anterior. B) Obturador quirúrgico de etilenovinil-acetato. C) Defecto maxilar de hemimaxilectomía por abordaje Weber-Ferguson. D) Obturador quirúrgico colocado. 
Rev Mex Cir Bucal Maxilofac. 2021;17 (1): 39-47

Figura 2:

Caso 2. A) Lesión tumoral erosiva de encía y proceso alveolar maxilar izquierdo correspondiente a carcinoma

epidermoide de células escamosas. B) Obturador quirúrgico a base de metilmetacrilato termocurable y retenedores de alambre.

C) Defecto maxilar de hemimaxilectomía por abordaje Weber-Ferguson. D) Obturador quirúrgico colocado conteniendo apósito quirúrgico.
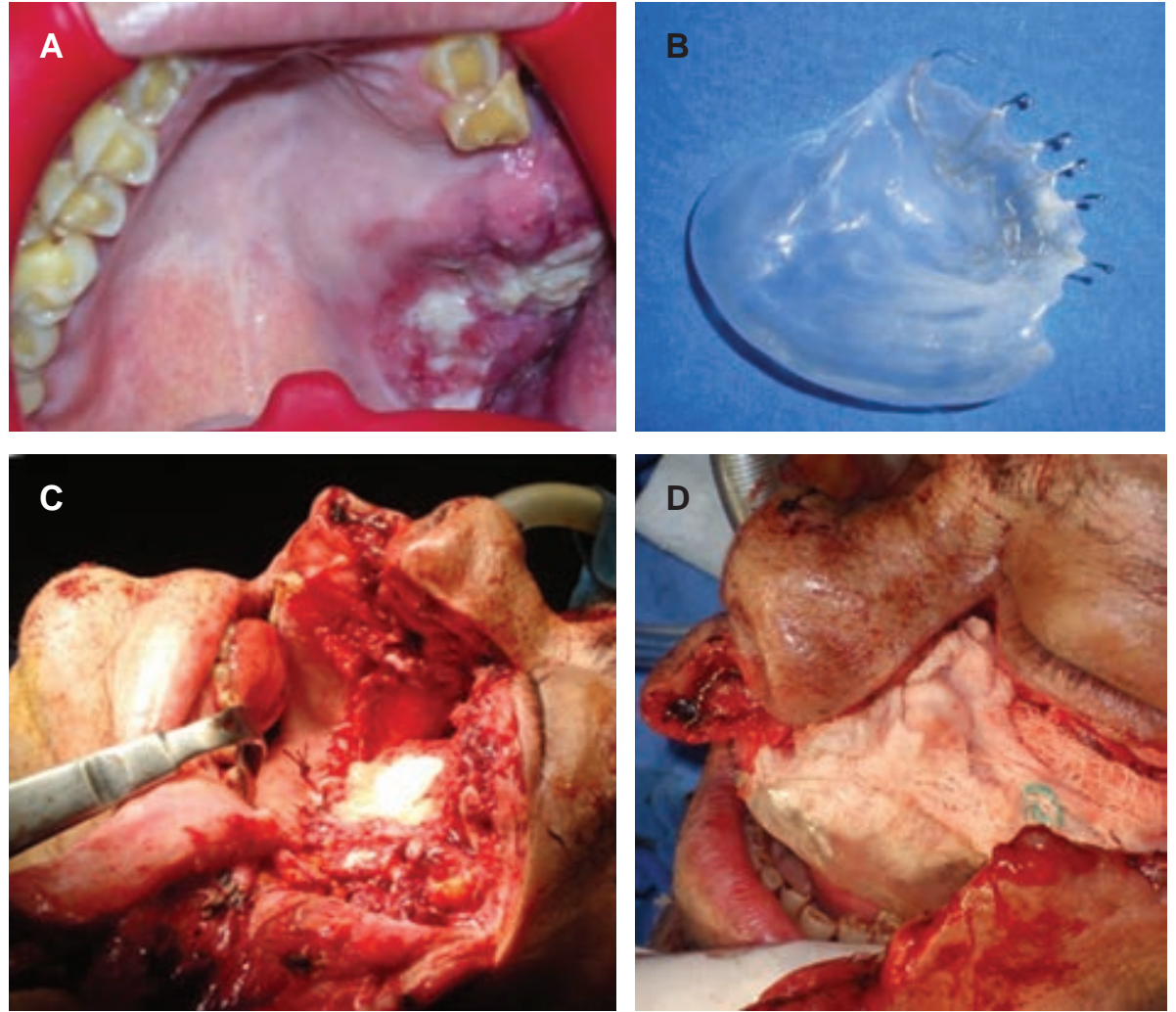
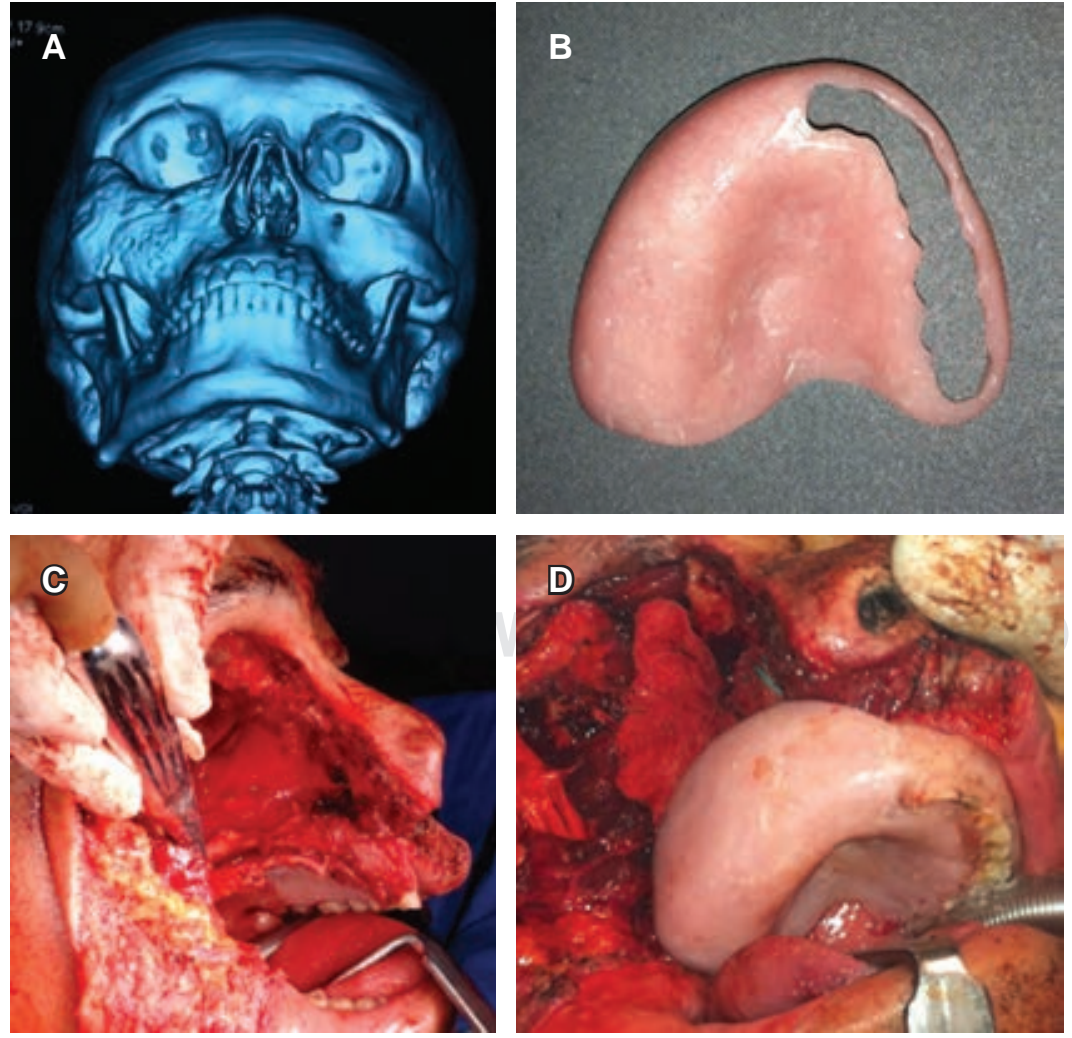

Figura 3:

Caso 3. A) Imagen de tomografía con reconstrucción $3 \mathrm{D}$ que muestra lesión osteoblástica en maxilar y cigomático derechos con afección de piso y pared lateral de orbita correspondiente a osteosarcoma. B) Obturador quirúrgico fenestrado a base de metilmetacrilato termocurable. C) Defecto de hemimaxilectomía y exenteración orbitaria derechas. D) Obturador quirúrgico colocado conteniendo apósito quirúrgico. 
Figura 4:

Caso 4. A) Lesión tumoral exofítica en encía y proceso alveolar maxilar en zona anterior y posterior izquierda correspondiente a carcinoma

epidermoide. B) Obturador quirúrgico dentado y fenestrado a base de metil-metacrilato

termocurable. C) Defecto maxilar de hemimaxilectomía por abordaje Weber-Ferguson.

D) Obturador quirúrgico colocado con aplicación de acondicionador de tejidos.
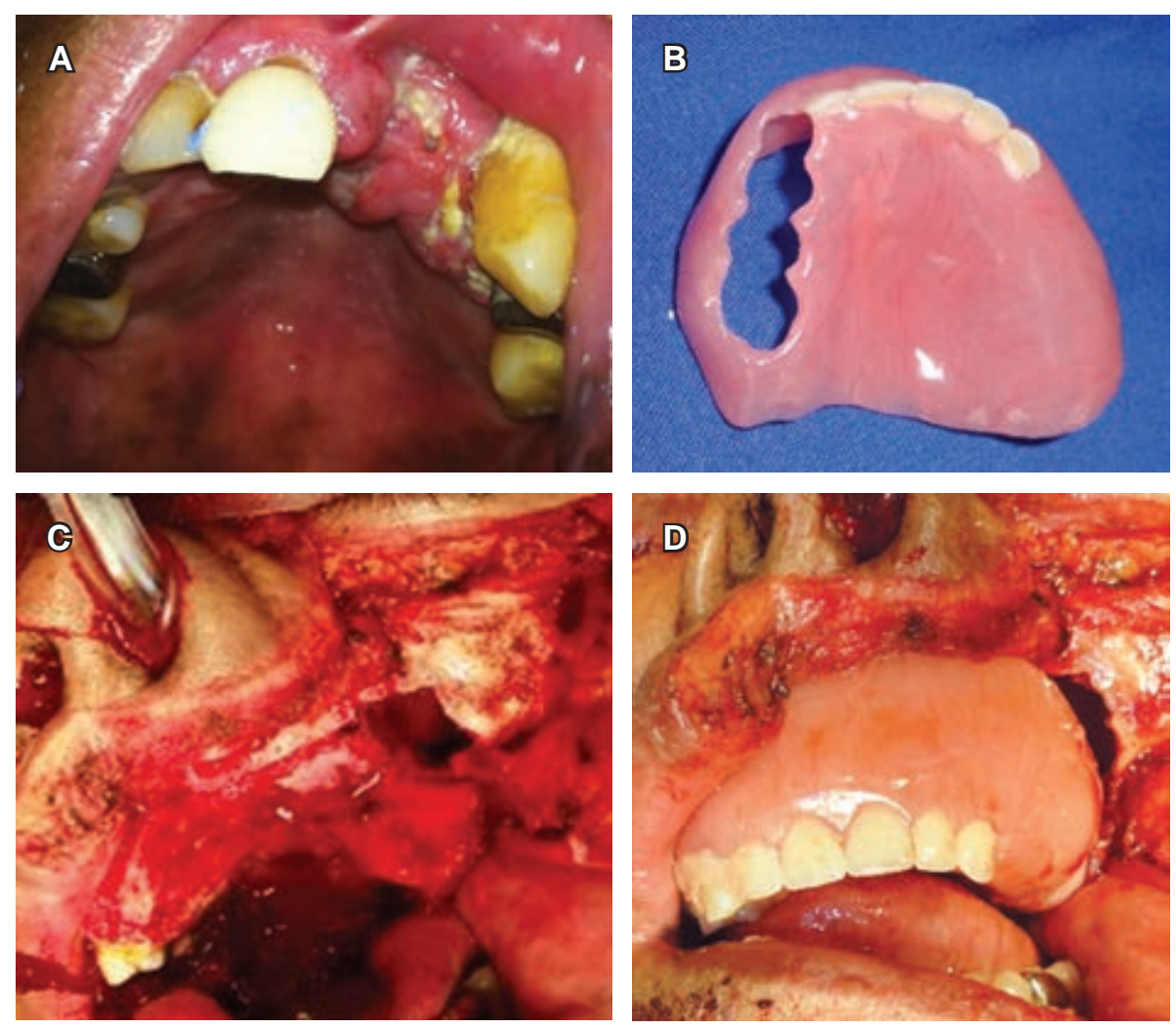
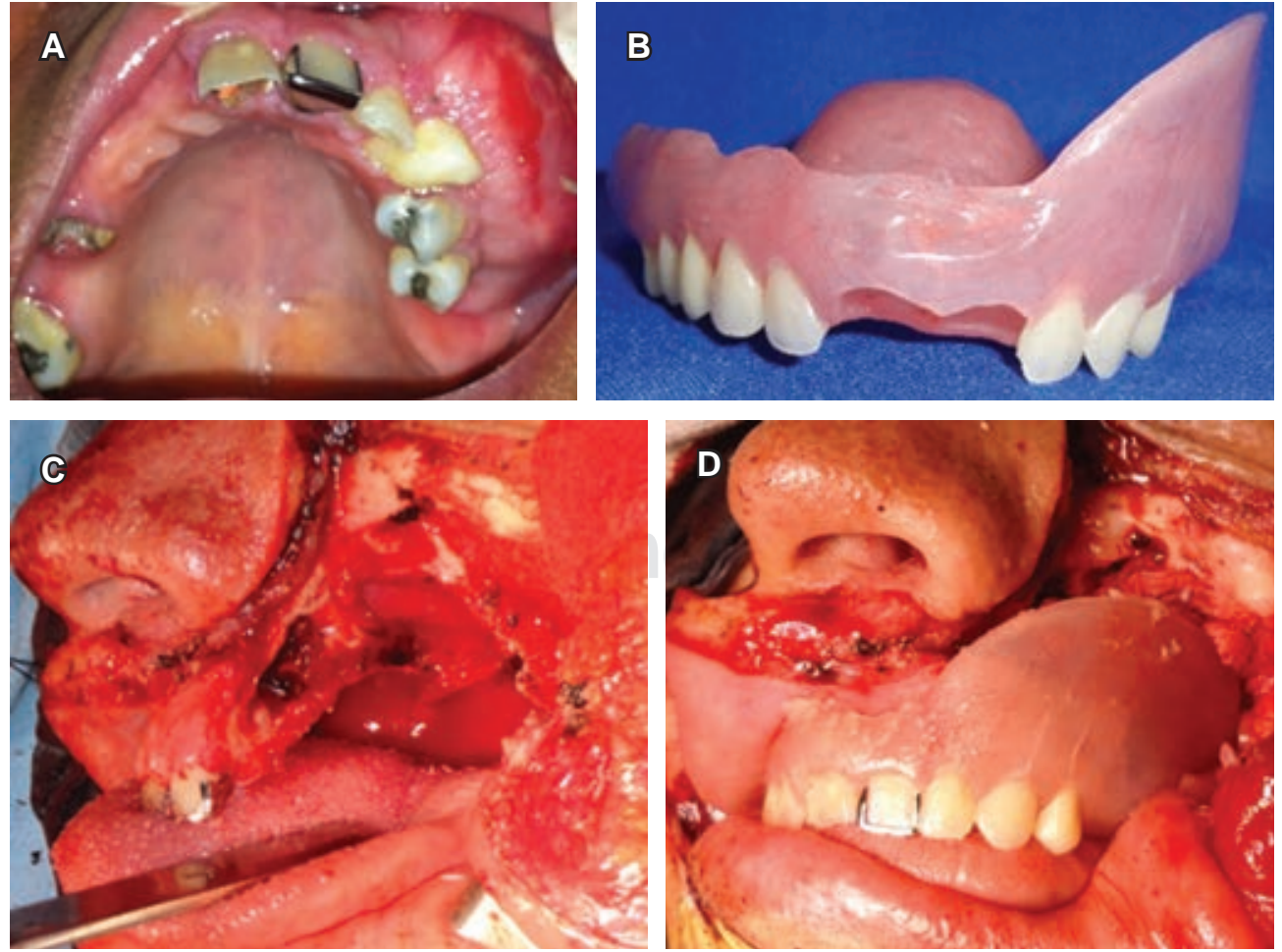

Figura 5:

Caso 5. A) Lesión

tumoral expansiva de encía y proceso alveolar maxilar izquierdo correspondiente a carcinoma primario intraóseo. B) Obturador quirúrgico dentado y fenestrado a base de metil-metacrilato termocurable. C) Defecto maxilar de hemimaxilectomía izquierda por abordaje Weber-Ferguson. D) Colocación de obturador quirúrgico con aplicación de acondicionador de tejidos y soportando apósito quirúrgico. 
toma impresiones previas a la cirugía con hidrocoloide irreversible y se decide diseñar y elaborar un obturador quirúrgico a base de etileno-vinil-acetato (EVA) de calibre 0.80 , esto debido a que el paciente tenía presentes todos los dientes del lado contralateral y nos permiten una adecuada retención por compresión para este tipo de obturador (Figura 1).

\section{Caso 2}

Hombre de 65 años con diagnóstico de carcinoma epidermoide de encía superior izquierda moderadamente diferenciado, con infiltración al seno maxilar. Después de la protocolización del caso, se programa para resección quirúrgica mediante hemimaxilectomía izquierda. El equipo de prótesis maxilofacial toma impresiones previas a la cirugía con hidrocoloide irreversible y se decide diseñar y elaborar un obturador quirúrgico a base de metil-metacrilato termocurable con retenedores de alambre labrados, mediante retenedores con punta de bola calibre 0.028 y retenedores de alambre de wipla de media caña calibre \#17, gracias a las zonas retentivas que presentan los dientes presentes que permiten colocar este tipo de retenedores (Figura 2).

\section{Caso 3}

Hombre de 45 años con diagnóstico de osteosarcoma maxilar derecho de bajo grado, con extensión al hueso cigomático, piso y pared lateral de órbita del mismo lado. Después de la protocolización del caso, se programa para resección quirúrgica mediante hemimaxilectomía de supraestructura derecha y exenteración orbitaria. El equipo de prótesis maxilofacial toma impresiones previas a la cirugía con hidrocoloide irreversible, se decide diseñar y elaborar un obturador quirúrgico a base de metil-metacrilato termocurable fenestrado para permitir el paso de los dientes que se van a conservar, esto debido a que el paciente presentaba bruxismo y no permitía colocar retenedores de alambre por futura sensación de cuerpo extraño a la oclusión durante la masticación. El obturador se coloca con ayuda de un acondicionador de tejidos temporal de tipo blando de mediana duración (COE-SOFT ${ }^{\mathrm{TM}} \mathrm{GC}$ America Inc.) para mejorar la retención y adaptación del mismo (Figura 3).

\section{Caso 4}

Mujer de 69 años con diagnóstico de carcinoma epidermoide de encía superior antero-izquierda bien diferenciado sin infiltración al seno maxilar. Después de la protocolización del caso, se programa para resección quirúrgica mediante hemimaxilectomía izquierda. El equipo de prótesis maxilofacial toma impresiones previas a la cirugía con hidrocoloide irreversible y se decide diseñar y elaborar un obturador quirúrgico a base de metil-metacrilato termocurable con dientes anteriores y fenestrado para permitir el paso de los dientes que se van a conservar en el lado contralateral; en este caso se decide colocar dientes protésicos anteriores, para mejorar la estética de la paciente, ya que era un aspecto que le preocupaba en exceso, estos dientes se deben colocar en oclusión mínima para no afectar el proceso de cicatrización del defecto. El obturador se coloca con ayuda de un acondicionador de tejidos temporal de tipo blando de mediana duración (COE-SOFT ${ }^{\mathrm{TM}} \mathrm{GC}$ America Inc.) para mejorar la retención y adaptación del mismo (Figura 4).

\section{Caso 5}

Mujer de 42 años con diagnóstico de carcinoma de células escamosas intraóseo primario moderadamente diferenciado del maxilar en la región posterior izquierda con infiltración al seno maxilar. Después de la protocolización del caso, se programa para resección quirúrgica mediante hemimaxilectomía izquierda. El equipo de prótesis maxilofacial toma impresiones previas a la cirugía con hidrocoloide irreversible y se decide diseñar y elaborar un obturador quirúrgico a base de metilmetacrilato termocurable con dientes protésicos posteriores del lado derecho, fenestrado en la zona anterior para permitir el paso de los dos centrales superiores que se conservarán. En este caso, se decide colocar dientes protésicos posteriores en el lado sano, debido a que la paciente no puede alimentarse de manera correcta por la ausencia de éstos, con esto mejoramos la estabilidad del obturador y la alimentación del caso. El obturador se coloca con ayuda de un acondicionador de tejidos temporal de tipo blando de mediana duración (COESOFT ${ }^{\mathrm{TM}} \mathrm{GC}$ America Inc.) para mejorar la retención y adaptación del mismo (Figura 5).

\section{DISCUSIÓN}

Los defectos quirúrgicos como resultado de la remoción de neoplasias malignas en la región maxilar varían, desde pequeñas perforaciones del paladar, hasta la completa remoción de estructuras anatómicas 
importantes. Los defectos, sin importar el tamaño, producen alteraciones funcionales importantes $y$, ocasionalmente, llegan a producir alteraciones faciales cosméticas. El habla será hipernasal, la masticación se verá comprometida y habrá dificultad para la deglución, así como comunicación de alimento hacia la cavidad nasal.

El obturador quirúrgico (o inmediato) restablece la separación física entre las cavidades oral y nasal. Muchos pacientes pueden ser rehabilitados exitosamente restaurando el habla normal, la deglución y mejorando significativamente su apariencia. Un obturador quirúrgico es imperativo en las resecciones del maxilar, para esto se requieren modelos de estudio y de trabajo que le permiten al cirujano planear la resección y al protesista maxilofacial el diseño y elaboración del aparato protésico. ${ }^{6}$

En nuestra experiencia, el material ideal para la toma de impresiones prequirúrgicas para la elaboración de obturadores inmediatos es el hidrocoloide irreversible, como se realizó en los casos presentados, así como en las investigaciones realizadas por Park, Naveen, Shambharkar y Farias, ya que este material permite reproducir de manera precisa los detalles de las estructuras que se conservarán, así como de la lesión tumoral a resecar, tomando en cuenta que no necesitamos un material más preciso debido a los cambios que se presentarán por el crecimiento de la neoplasia y durante su resección. 8,17,21,23

Para el diseño del obturador, se deben tomar en cuenta muchos aspectos como son, el tamaño y extensión de la neoplasia, la cantidad y calidad de los dientes que se van a conservar, el diseño de la resección maxilar, la posibilidad de tratamientos adyuvantes posteriores a la cirugía (quimioterapia o radioterapia), la presencia de trismus preoperatorio, dientes antagonistas, habilidad del paciente para su inserción y retiro, y algo muy importante, el tiempo con el que dispongamos previo a la cirugía para la elaboración del obturador.

Tomando en cuenta estos factores, en el caso número 1 se decidió elaborar el obturador mediante la técnica de etileno-vinil-acetato (EVA), similar a lo realizado por Fandiño y Ugalde, ${ }^{6,24}$ ya que es una técnica sencilla, rápida y de bajo costo, nos provee una sujeción adecuada sobre las coronas de los dientes remanentes por compresión, además de ser fácilmente modificable durante el acto quirúrgico. Otra razón por la que se recomienda el uso de este diseño, es cuando tenemos poco tiempo para la elaboración y la cirugía es urgente o no hubo previa interconsulta con el protesista maxilofacial, ya que nos lleva un tiempo muy reducido el diseñarlo y fabricarlo. En algunos casos se puede agregar metilmetacrilato autocurable color rosa y color diente en el interior del obturador para mejorar la estética del paciente en el postoperatorio como lo mostró Murillo. ${ }^{9}$

Este tipo de obturador, no se recomienda adaptarlo como transicional de mediano plazo, ya que por las características del material, tiende a colonizarse por microorganismos micóticos y bacterianos.

En el caso 2 se decidió por un obturador a base de una placa de metil-metacrilato termocurable con aleta de extensión hacia el defecto y con retenedores labrados a base de alambre de wipla y alambre con punta de bola, similar a lo realizado por Luthra y Alhajj, ${ }^{7,11}$ esto debido a la anatomía de los dientes que se van a conservar, ya que nos dan una adecuada retención; además, la prótesis se puede mantener como obturador transicional por tiempo indefinido después del desempaquetamiento a los cinco días de postoperatorio, esto debido a que el paciente recibiría tratamiento concomitante de quimioterapia y radioterapia, lo que no nos permitiría tomar impresiones para elaborar un nuevo obturador, por los efectos adversos de estos tratamientos (mucositis y xerostomía), así como un defecto cambiante por retraso en la reepitelización y falta de cierre del mismo por los tratamientos concomitantes.

En el caso número 3 se decidió elaborar un obturador a base de una placa de metil-metacrilato termocurable con aleta de extensión hacia el defecto y un fenestrado que permite el paso de los dientes remanentes y su adaptación en el cuello de los mismos, esto debido a que el paciente presentaba un bruxismo severo y cualquier aditamento de retención a base de alambre o acetato provocaría interferencia oclusal a la masticación; asimismo, por el tamaño del defecto planeado, la histología de la neoplasia y el posterior tratamiento adyuvante con QT, se planeó que este obturador se adaptara como transicional durante el tratamiento de QT. En la literatura consultada, no se encontró un caso similar utilizando este diseño, por lo que en este artículo se propone su diseño para su uso en los casos que se crea conveniente, con el nombre de «obturador fenestrado». Para mejorar la retención del obturador, se sugiere el uso de acondicionador de tejidos blandos para adaptarse al fenestrado y a los cuellos de los dientes.

En el caso 4 se decidió realizar un obturador a base de una placa de metil-metacrilato termocurable 
con aleta de extensión hacia el defecto, un fenestrado para la retención de los dientes remanentes, ya que al igual que el caso número 2, la oclusión de la paciente estaba muy cerrada y no permitía colocar retenedor de alambre o acetato; además de la colocación de dientes protésicos en la zona anterior, esto debido a que a la paciente le preocupaba mucho el aspecto estético postquirúrgico, decidiendo esta estrategia para evitar depresión postoperatoria por afección severa de su estética facial, similar a lo realizado por Patil y Shambharkar. ${ }^{10,20,21}$ De manera tradicional, se ha evitado la colocación de dientes protésicos en el obturador quirúrgico porque se cree que puede provocar lesiones en los tejidos que se encuentran en etapa de cicatrización, provocando un retraso en la misma, en especial en aquéllos que recibirán radioterapia adyuvante como comenta Farias, ${ }^{23}$ aunque no existe evidencia científica contundente que demuestre este dicho.

En el caso 5 se realizó un obturador a base de una placa de metil-metacrilato con aleta de extensión hacia el defecto, fenestrado en la zona de los OD 11, 21 y 17 para permitir el paso de los dientes que se van a conservar y la colocación de dientes protésicos en la zona anterior y en la zona posterior derecha, esto con el fin de mejorar la estética de la paciente, y principalmente para mejorar la masticación del lado contralateral al defecto, ya que la paciente no tiene órganos dentarios funcionales en la porción del maxilar que se va a conservar y debido a una pérdida de peso importante y a la posibilidad de recibir tratamiento concomitante, se decidió este diseño con dientes, para mejorar la alimentación de la paciente. Como se ha mencionado antes, para mejorar el ajuste del obturador, se debe emplear un acondicionador de tejidos blando de mediano plazo, al igual que para disminuir el impacto oclusal de los dientes protésicos sobre los tejidos del defecto que entrarán en proceso de cicatrización. El agregar dientes protésicos al obturador quirúrgico tiene efectos positivos en varios aspectos, como es mejorar el soporte de los tejidos blandos faciales y bucales, rehabilitar lo más pronto posible la masticación evitando dietas líquidas o blandas, evita afectar la deglución al poder ingerir alimentos de consistencias más sólidas, disminuye las alteraciones a nivel de la articulación temporomandibular y, sobre todo, mejora de manera importante la fisonomía y estética facial y dental, contribuyendo de manera positiva en el ánimo del paciente, y tiene un efecto psicológico positivo, como lo menciona Patil. ${ }^{10}$

\section{CONCLUSIÓN}

El principal objetivo de la prótesis maxilofacial como especialidad odontológica en el tratamiento de pacientes con neoplasias de la región maxilofacial es el de mejorar su calidad de vida, y en tratamientos que incluyen resecciones maxilares, el uso de obturadores quirúrgicos cumple con este fin al reestablecer las funciones del habla, la masticación y la deglución, así como el brindar soporte a los tejidos blandos para evitar que se presenten asimetrías faciales, bridas cicatrizales o retracciones de tejidos que afectarían de manera importante la estética, teniendo un efecto negativo en la autoestima del paciente.

El uso de obturadores quirúrgicos es esencial para la rehabilitación inmediata y futura de las funciones orales y estéticas del paciente, además de que es un complemento del tratamiento quirúrgico que debe ser adaptado de manera rutinaria por todos los cirujanos del área de cabeza y cuello para impactar de manera positiva en la recuperación del paciente.

\section{REFERENCIAS}

1. Bray F, Ferlay J, Soerjomataram I, Siegel RL, Torre LA, Jemal A. Global cancer statistics 2018: GLOBOCAN estimates of incidence and mortality worldwide for 36 cancers in 185 countries. CA Cancer J Clin. 2018; 68: 394-424.

2. Granados M, Aguilar JL, Luna K, Maldonado F, Lavin A. El tratamiento multidisciplinario del cáncer de las vías aerodigestivas superiores. Cancerología. 2007; 2: 27-38.

3. Beumer J, Marunick MT, Esposito SJ. Maxillofacial rehabilitation: prosthodontic and surgical management of cancer-related, acquired, and congenital defects of the head and neck. 3a ed. United States: Quintessence Pub; 2011.

4. Forna DG, Sulea D, Costan VV, Popescu E. Conventional maxillary reconstruction using service obturators. Romanian J Oral Rehab. 2016; 8: 32-39.

5. Granados García M, Arrieta Rodríguez OG, Hinojosa Gómez J. Tratamiento del cáncer: oncología médica, quirúrgica y radioterapia. México: Editorial El Manual Moderno; 2016.

6. Fandiño TLA, López PRA. Elaboración de obturador quirúrgico (prótesis inmediata) en un paciente con hemimaxilectomía por carcinoma epidermoide. Rev ADM. 2001; 58 (6): 220-228.

7. Alhajj MN, Ismail IA, Khalifa N. Maxillary obturator prosthesis for a hemimaxillectomy patient: a clinical case report. Saudi J Dent Res. 2016; 7: 153-159.

8. Park KT, Kwon HB. The evaluation of the use of a delayed surgical obturator in dentate maxillectomy patients by considering days elapsed prior to commencement of postoperative oral feeding. $\mathrm{J}$ Proshet Dent. 2006; 96: 449-453.

9. Murillo HSA, López PRA. Obturador temporal, una alternativa de tratamiento para defectos maxilares adquiridos en un paciente infantil: Reporte de un caso clínico. Rev ADM. 2008; 65 (2): 88-96.

10. Patil PG. Surgical obturator duplicating original tissue-form restores esthetics and function in oral cancer. World J Stomatol. 2013; 2 (4): 97-102. 
11. Luthra RP, Gupta R, Gupta S. Fabrication of a surgical obturator in a patient of squamous cell carcinoma with reduced mouth opening. IJHS. 2016; 3 (2): 537-540.

12. The glossary of prosthodontic terms: ninth edition. J Prosthet Dent. 2017; 117 (5S): e1-e105.

13. Sarin S, Gupta R, Luthra RP, Ahirrao R, Sharma V. History of evolution of palatal obturators. J Adv Med Dent Scie Res. 2015; 3 (2): 46-53.

14. Keyf F. Obturator prostheses for hemimaxillectomy patients. J Oral Rehabil. 2001; 28 (9): 821-829.

15. Joshi AD, Sukre S, Bhandari AJ, Bandewar A, Dhope S, Gangadhar SA. Prosthodontic step wise rehabilitation of a patient with polymorphous low-grade adenocarcinoma (PLGA) in maxilla a case report. Pravara Med Rev. 2016; 8 (3): 29-32.

16. Duggal A, Arora A, Duggal P, Gill TK. Obturator prosthesis for rehabilitation of acquired maxillary defect $A$ case report IJCDC. 2016; 6 (2): 801-803.

17. Naveen BH, Kashinath KR, Ravi KN, Kalavathi SD. Preplanned surgical obturator prosthesis A boon for rehabilitation. J Dent Sciences and Research. 2011; 2 (2): 50-55.

18. Sullivan M, Gaebler C, Beukelman D, Mahanna G, Marshall J, Lydiatt $D$ et al. Impact of palatal prosthodontic intervention on communication performance of patients' maxillectomy defects: a multilevel outcome study. Head Neck. 2002; 24 (6): 530-538.

19. Turkaslan S, Baykul T, Aydin MA, Ozarslan MM. Influence of immediate and permanent obturators on facial contours: a case series. Cases Journal. 2009; 2 (1): 1-5.

20. Patil PG. New technique to fabricate an immediate surgical obturator restoring the defect in original anatomical form. $J$ Prosthodont. 2011; 20 (6): 494-498.

21. Shambharkar VI, Puri SB, Patil PG. A simple technique to fabricate a surgical obturator restoring the defect in original anatomical form. J Adv Prsothodont. 2011; 3: 106-109.

22. Kar S, Tripathi A. Treatment outcome with delayed maxillary obturator prosthesis: case series of four patients. J Prosthodont. 2016; 25 (2): 174-177.

23. Farias A, Hegde C, Krishnaprasad D. A simplified technique to make an immediate surgical obturator for a maxillectomy patient. $\mathrm{J}$ Interdiscip Dentistry. 2013; 3 (3): 125-128.

24. Ugalde Ojeda ML, Granados García M. Obturadores para defectos palatinos: Un nuevo método de elaboración. Rev Inst Nal Cancerol. 1998; 44: 15-18. 